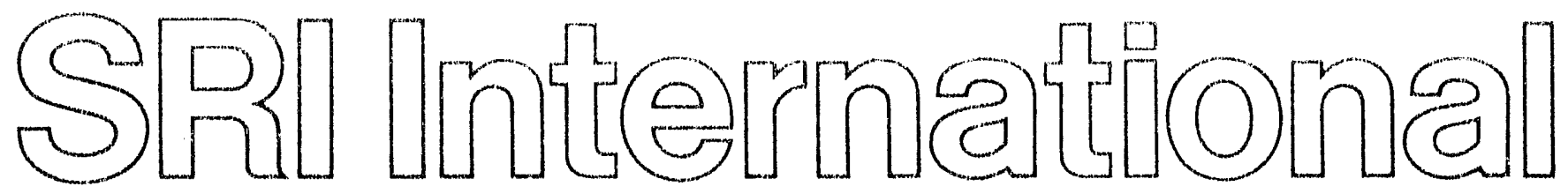

\title{
HYDROTHERMAL PRETREATMENT OF COAL
}

\author{
Bock Loo and David S. Ross \\ Organic and Inorganic Chemistry Department
}

Covering the Period July 16 to October 15,1990

Prepared for:

U.S. Department of Energy

Pittsburgh Energy Technology Center

P.O. Box 10940, MS 921-165

Pittsburgh, PA 15236

Contract No. DE-AC22-89PC89880

Approved:

David M. Golden

Laboratory Director

Chemistry Laboratory

\section{DISCLAIMER}

This report was prepared as an account of work sponsored by an agency of the United States Government. Neither the United States Government nor any agency thereof, nor any of their employees, makes any warranty, express or implied, or assumes any legal liability or responsibility for the accuracy, completeness, or usefulness of any information, apparatus, product, or process disclosed, or represents that its use would not infringe privately owned rights. Referpece herein to any specific commercial product, process, or service by trade name, trademark, manufacturer, or otherwise does not necessarily constitute or imply its endorsement, recommendation, or favoring by the United States Government or any agency thereof. The views and opinions of authors expressed herein do not necessarily state or reflect those of the United States Government or any agency thereof. 


\begin{abstract}
During this period we studied the effects of $350^{\circ} \mathrm{C}$ treatment on Argonne-supplied Wyodak coal with the n-alkane undecane in place of water in both $30 \mathrm{~min}$ and $5 \mathrm{hr}$ experiments. Undecane was chosen because it has a critical temperature of $363^{\circ} \mathrm{C}$ (calculated), similar to that for water, and is present as a liquid phase at conditions. We anticipated results little different from those from the simple thermal treatment (coal under $\mathrm{N}_{2}$ ) at the same conditions. In that case we had found the production of volatiles, which were subsequently monitored by field ionization mass spectrometry (FIMS).

We found the undecane treatment, however, affected the coal differently. The volatiles production was suppressed substantially after 30 min treatment, and the product coal after $5 \mathrm{hr}$ showed virtually no volatility. It appears that the alkane encourages regressive chemistry, and our rationale at present involves its suppressing migration of reactive phenolic fragments from the matrix, and their subsequent irreversible reincorporation. The key element in the process we suggest is the net conversion of labile coalO-Ar bonds to strong coal-Ar bonds.
\end{abstract}




\section{INTRODUCTION}

We are examining the effects of hydrothermal treatment (liquid water $/ 150^{\circ}-350^{\circ} \mathrm{C}$ ) on the structure and reactivity of Wyodak coal. The studies are conducted in small bomb reactors with quartz inserts, under nitrogen, and with sufficient water added to ensure the presence of liquid water at treatment conditions. The treatment periods are $30 \mathrm{~min}$ and 5 $\mathrm{hr}$, and the coal is "dried" before use by holding it overnight at 1 torr and $60^{\circ} \mathrm{C}$. About $20 \%$ of the coal mass is lost, presumably as water, and all values presented are on the basis of such "dried" samples.

Thermal treatments (runs with no added water) are conducted routinely as controls, and during this research period additional control runs were performed with the normal alkane undecane $\left(\mathrm{C}_{11} \mathrm{H}_{24}\right)$ replacing water as medium. Undecane was chosen because it is an essentially nonreducing, reasonably unreactive organic medium with a critical temperature estimated at $363^{\circ} \mathrm{C}$ (Lydersen, 1955), similar to that of water.

Our diagnostics include extensive field ionization mass spectrometry (FIMS) analysis. This procedure involves heating $1.0-1.5 \mathrm{mg}$ samples of product at $2.5^{\circ} \mathrm{C} / \mathrm{min}$ from ambient to $500^{\circ} \mathrm{C}$ and taking spectra of the volatiles at $20^{\circ}-30^{\circ}$ intervals. Thermal gravimetric analyses (TGA) were also conducted at the same heating rate.

Initial studies at $150^{\circ}$ and $250^{\circ} \mathrm{C}$ over $30 \mathrm{~min}$ showed that little change in the coal. Subsequent work at $350^{\circ} \mathrm{C}$ showed that in contrast there was clear change after $30 \mathrm{~min}$ treatment, and substantially more following exhaustive $5 \mathrm{hr}$ treatment.

\section{PAST RESULTS}

In our last quarterly report we discussed the differences we found in $30 \mathrm{~min}$ and 5 $\mathrm{hr}$ treatments (Ross, et al., 1990a). We found that thermal treatment at both $30 \mathrm{~min}$ and 5 $\mathrm{hr}$, and $30 \mathrm{~min}$ hydrothermal treatment, reduced the oxygen content of the coal by about $30 \%$. However in the $5 \mathrm{hr}$ hydrothermal treatment the coal lost another $15 \%$ of its oxygen. This result suggest that water promotes O-loss chemistry, most likely acid catalyzed dehydroxylation. In concert with this activity, the aqueous conditions promote the deposition of a tar on the walls of the quartz inserts corresponding to 5-7\% of the starting coal, and the shift from $30 \mathrm{~min}$ to $5 \mathrm{hr}$ yields a tar that is more volatile and has a slightly increased molecular weight. No deposited tar is seen at thermal conditions.

FIMS data showed significant reductions in the dihydroxybenzenes content (primarily catechols) of the FIMS-volatiles following both $30 \mathrm{~min}$ thermal and $30 \mathrm{~min}$ 
hydrothermal treatment. The phenol values, on the other hand, remained about the same. At $5 \mathrm{hr}$ hydrothermal conditions, both the phenols and catechols were almost fully eliminated from the FIMS volatiles, consistent with both hydrolysis and the increased loss of oxygen.

In solvent swelling studies, both the thermal and hydrothermal treatments yielded products with a decreased swelling ratio, but the ratio for the product from the aqueous treatment was lower than that from thermal treatment. This result was a surprise, since we had expected the presence of water to decrease the degree of thermal crosslinking.

\section{CURRENT RESULTS}

The solvent swelling results prompted studies with undecane replacing water as the treatment medium. In this work we sought a probe of the crosslinking chemistry operative at our conditions, and an understanding of the relationship between crosslinking and the mobility of the tars and/or tar precursors.

Table 1 contains $\mathrm{H} / \mathrm{C}$ and $\mathrm{O} / \mathrm{C}$ data for recovered sample from several conditions including undecane $/ 30 \mathrm{~min}$. Undecane $/ 5 \mathrm{hr}$ data were not available at the time of writing. The undecane $/ 30 \mathrm{~min} \mathrm{H} / \mathrm{C}$ value shows that there is no significant incorporation of the alkane into the recovered coal at least for that period of treatment. The increase in O-loss at hydrothermal $/ 5 \mathrm{hr}$ reflects the "stripping" of arenols from the coal through the large scale hydrolysis discussed in our last report. Notable here, however, is the somewhat higher $\mathrm{O} / \mathrm{C}$ ratio for undecane $/ 30 \mathrm{~min}$, indicating that at those conditions some oxygen is retained.

Consistent with these data are results in Figure 1, which is derived from Figure 4 of our last quarterly report (Ross, et al., 1990a). The figure shows the FIMS profiles for $\mathrm{m} / \mathrm{e}=94$ and $\mathrm{m} / \mathrm{e} 110$, which correspond respectively to phenol and dihydroxybenzenes. The $\mathrm{C}_{1^{-}}, \mathrm{C}_{2^{-}}$, and $\mathrm{C}_{3}$-derivatives showed similar profiles. We noted last time that the figure demonstrated the sensitivity of the dihydroxybenzenes or their thermolytic precursors to the thermal and hydrothermal pretreatment, and showed the extensive arenol loss at the extended hydrothermal conditions. We noted further that the significant loss of dihydroxybenzene signal under thermal conditions to larger fragments at those conditions.*

\footnotetext{
* We neglected to discuss another interesting feature of the data. The figure shows dual desorption paths primarily for phenols indicating a shift of significant fractions to evolution at lower temperatures. This phenomenon is similar to the split thermal desorption profiles reported by Malhotra and Haynes for the desorption of $t$-butylamine from several acid surfaces, and reflects the presence of both strongly and weakly bound substrate (1988). The shift seen here thus shows directly the formation of the arenols in the pretreatment, which are then not lost but rather retained via hydrogen bonding to the coal matrix.
} 


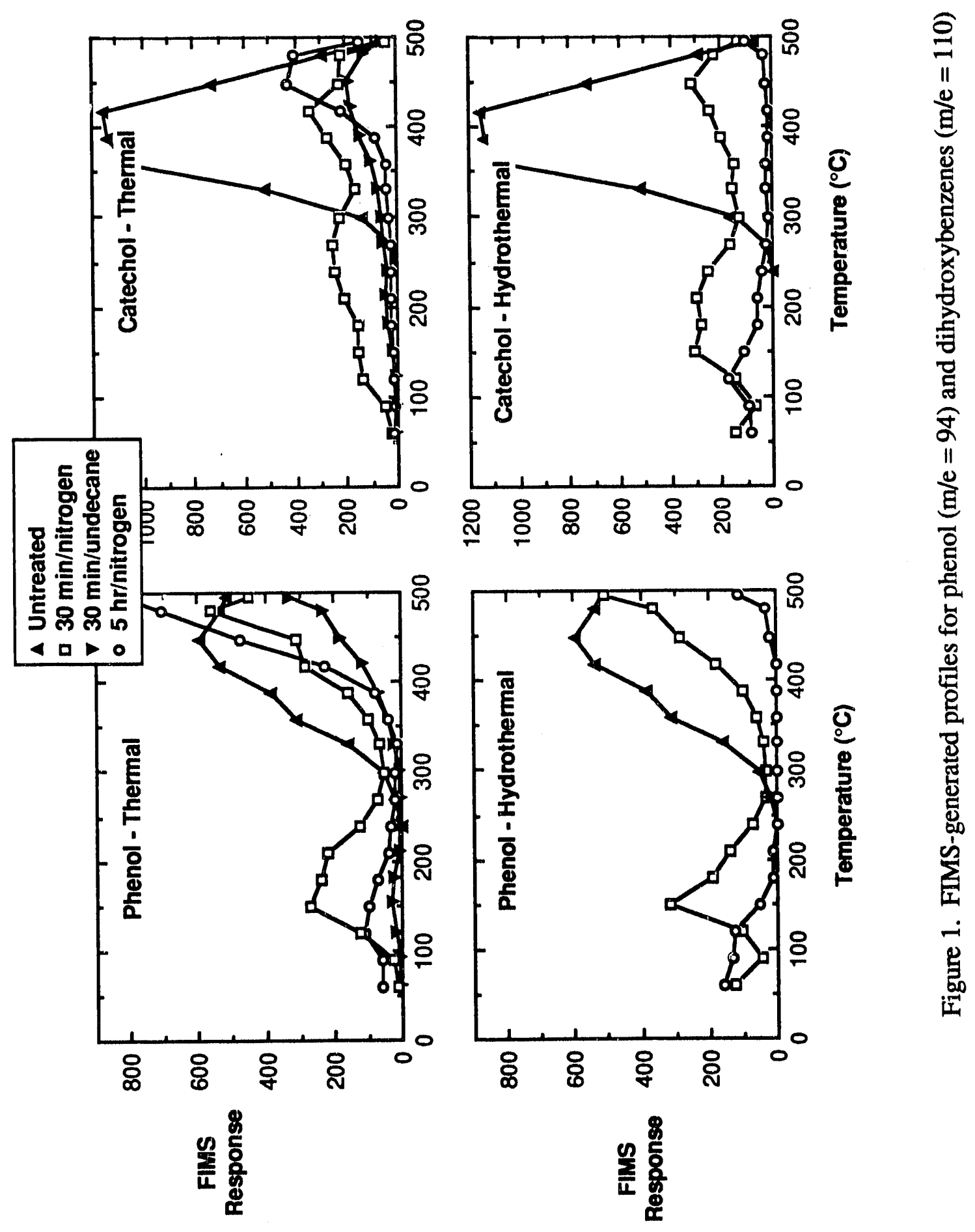


7 able 1

H/C and O/C Ratios of Product Wyodak Coals

Treatment Period

"Dried" Hydrothermal Thermal Undecane
Starting
Coal

$\mathrm{H} / \mathrm{C}$

$$
0.90
$$

$\begin{array}{ccccc}30 \mathrm{~min} & - & 0.82 & 0.83 & 0.81 \\ 5 \mathrm{hr} & - & 0.72 & 0.76 & -\end{array}$

$\mathrm{O} / \mathrm{C}^{\mathrm{a}}$

0.24

$\begin{array}{ccccc}30 \mathrm{~min} & - & 0.16 & 0.15 & 0.18 \\ 5 \mathrm{hr} & - & 0.12 & 0.16 & -\end{array}$

a. Oxygen determined by direct $\mathrm{O}$-analysis.

In the present figure we have added the phenol and catechol profiles for the results from our undecane/30 min runs, and complementary information is provided in Table 2. The figure shows that after just $30 \mathrm{~min}$, the response for both arenols is reduced to levels well below those for the thermal runs, a 70-80\% reduction as shown in the table. The FIMS data for the $5 \mathrm{hr}$ treatment, not shown for clarity, fall yet further below those. Clearly a large change in the thermolytic chemistry of these O-containing species takes place when undecane is present. Taken with the $\mathrm{O} / \mathrm{C}$ data in Table 1 , the results in the figure suggest that the arenols otherwise thermally released are, when the alkane is present, retained within the matrix and reincorporated.

These results are amplified by data shown in Figure 2, which displays FIMS ion count values as a function of sample ter incrature for samples for both $30 \mathrm{~min}$ and $5 \mathrm{hr}$ treatment. FIMS mass analysis begins at $\mathrm{m} / \mathrm{e} 48$, and so the signal is independent of the evolution of water and carbon dioxide. It reflects solely organic volatiles. For comparison the figure includes profiles for the untreated and the thermally- and hydrothermally-treated coal. The profile for the untreated coal displays generation of significant levels of 

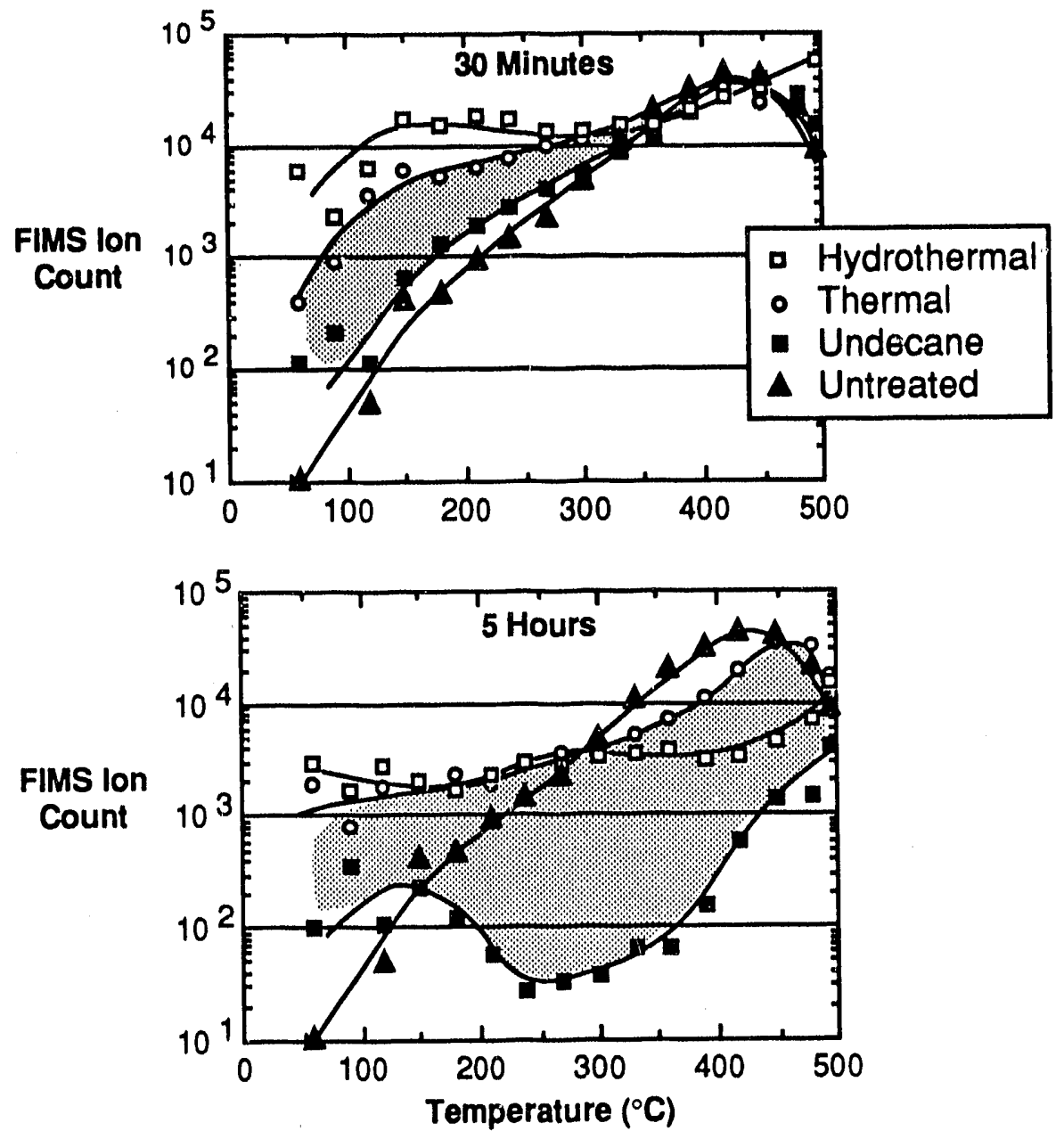

Figure 2. FIMS ion count values vs evaporation temperature for $30-\mathrm{min}$ and 5 -hr runs at $350^{\circ} \mathrm{C}$. The shaded areas reflect the loss of FIMS-volatiles from the addition of undecane. 
Table 2

Characteristics of the FIMS Volatiles

$\begin{array}{cccc}\text { Treatment Period } & \begin{array}{c}\text { "Dried" Hydrothermal Thermal } \\ \text { Starting } \\ \text { Coal }\end{array}\end{array}$

Phenol $^{\mathrm{a}}$

$\begin{array}{ccccc}- & 1.00 & - & - & - \\ 30 \mathrm{~min} & - & .87 & 1.11 & .31 \\ 5 \mathrm{hr} & - & .24 & .84 & <.1\end{array}$

Cateshol $^{\mathrm{a}}$

$\begin{array}{ccccc}30 \mathrm{~min} & - & .61 & .51 & .22 \\ 5 \mathrm{hr} & - & .17 & .24 & <.1\end{array}$

$\mathrm{I}_{\left.1 / 2 \mathrm{~S}^{\circ} \mathrm{C}\right)^{\mathrm{b}}}$

$\begin{array}{ll}- & 395-400\end{array}$

$30 \mathrm{~min}$

$340-350$

$377-395$

405

$5 \mathrm{hr}$

410

425

465

$\overline{\bar{M}}_{w} \mathrm{c}$

- 403-411

$30 \mathrm{~min}$

$410-421$

329-380

373

$5 \mathrm{hr}$

332

308

201

a. Values correspond to the relative areas under the profiles in Figure 1, relative to the respective responses for the "dried" samples.

b. The temperature at which one-half of the FIMS volatiles has passed into the instrument.

c. Weight average molecular weight.

volatiles above $350^{\circ} \mathrm{C}$, the temperature region where bond breaking within the bulk coal become significant. This behavior, discussed in our last report (Ross, et al., 1990a), shows that most of the volatiles from the coal are the result of bond breaking, and are not withir the coal as separate, preexisting material. The volatiles generated by the $30 \mathrm{~min}$ 
thermal treatment are apparent in the figure, as is the effect of added water. The latter are most likely the heavier end of the water-generated tar that is not sufficiently mobile to migrate fully from the matrix to the reactor walls.

The undecane results, however, are most revealing. 'The figure shows that the undecane/30 min profile falls considerably below the thermal profile, and is little different from that for the untreated coal. The shaded area represents the volatiles lost by the addition of the alkane to coal/ $/ \mathrm{N}_{2}$. For the $5 \mathrm{hr}$ treatments the results that process is carried to it limit. The shaded area in this case shows a virtual total loss of response over the entire temperature range. The product is a char.

In line with the Figure 1 data, therefore, the presence of the normal alkane medium in some manner fortifies the regressive or condensation chemistry taking place at elevated temperatures. Such a drastic change in behavior is not expected in view of the nominal change in behavior with the increase in the simple thermal treatment under $\mathrm{N}_{2}$ from $30 \mathrm{~min}$ to $5 \mathrm{hr}$. And while at present with no composition data we cannot speculate on the degree of incorporation of the medium into the coal, we would not expect an H-rich alkane to bring about extensive condensation." We conclude that the undecane results are the result of the chemistry of the coal components, and that the action of the medium is tied to elements of solvent compatibility and mass transfer, as described below.

\section{DISCUSSION}

The breadth of behavior we have seen in this work ranges from the total separation of tars from the coal when it is heated with liquid water, through changes in tar and volatiles activity with heating just under nitrogen, to the unexpected enhanced condensation with undecane present. A number of factors are in play here, and we suggest that the core elements include:

- The lignitic nature of lower rank coals, the thermolytic and hydrolytic chemistry of lignin-derived ethers, and the reactivity of the dihydroxybenzenes derived from such structures;

- The collapse of the coal structure in nonaccommodating media, affecting the migration of thermolytic fragments and the reaction kinetics, a

- The respective short and long lifetimes of O-Ar and C-Ar bonds.

\footnotetext{
* The extraction of the FIMS-volatile material by the alkane medium might be suggested as an explanation for these effects. That route seems unlikely, however, since alkanes are such poor solvents for all but the least polar coal fractions. Moreover the substantial increase in volatiles loss in shifting the treatment from $30 \mathrm{~min}$ to $5 \mathrm{hr}$ cannot be explained by simple extraction.
} 
The following discussion, an extension of our comments presented in an earlier report (Ross, et al, 1990b), seeks to reconcile all of our observations on the basis of these points

Scheme 1 presents a rationale for the findings over the range of conditions investigated. The first step is the thermal generation of arenols, augmented in the hydrothermal case by hydrolysis. The reactivity of the arenols and the dihydroxyarenes in particular then becomes significant, in competition with their migration from the reactive sites within the coal matrix.

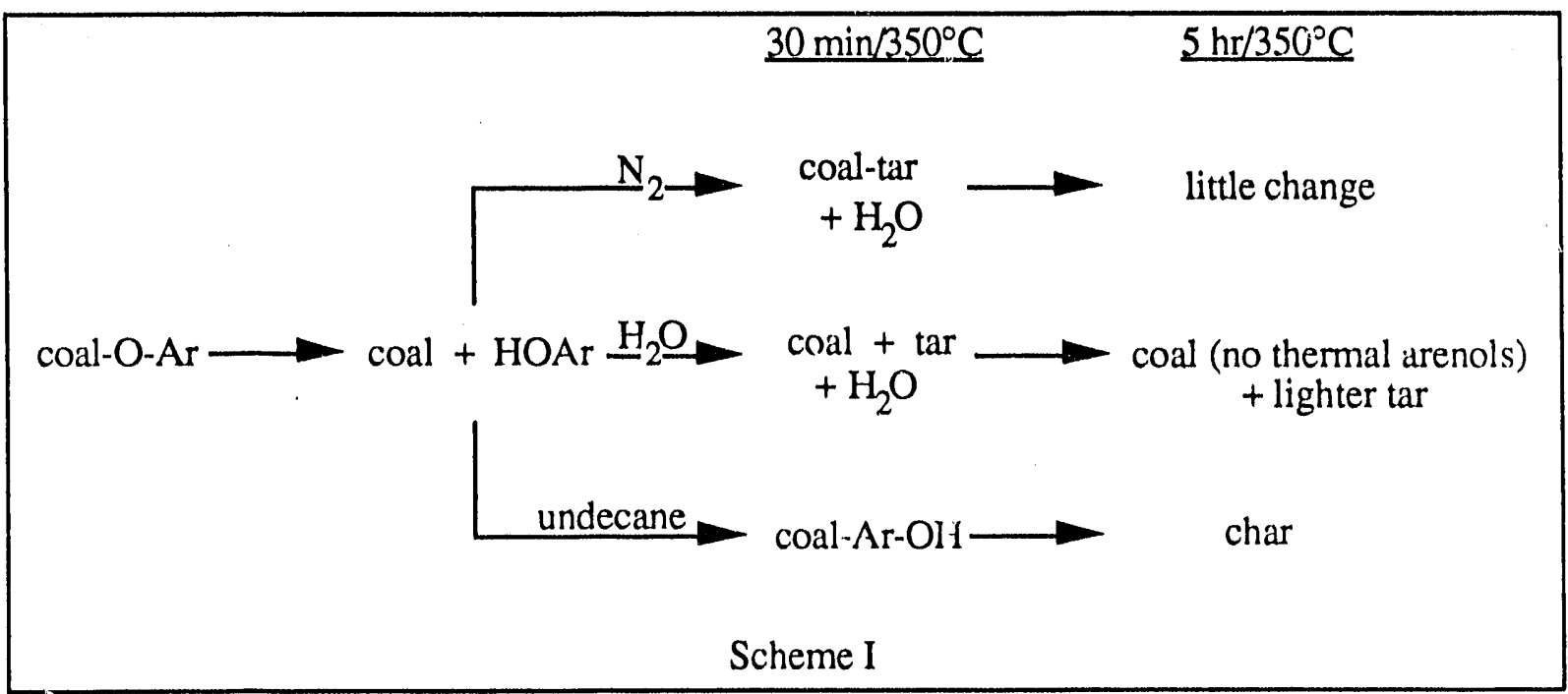

Thermal Treatment. In simple treatment under $\mathrm{N}_{2}$ the thermally generated fragments can migrate to some degree to other regions of the coal. They probably tend to concentrate at the mineral sites due to acid/base interactions, and at this point the recent results of Stein et al. can apply (1989). Their work showed that at $400^{\circ} \mathrm{C}$ dihydroxybenzenes are very reactive and undergo acid catalyzed chemistry. For catechol they found that water was formed from $15-20 \%$ of the consumed catechol, while the larger fraction underwent condensation to essentiaiiy "polycatechol," a nonvolatile residue where the oxygen was retained. In the case of coal, with distributions of clay and silica particles throughout the organic phase in sizes down perhaps to the nanometer level (Alien and VanderSande, 1984), the relative magnitudes of dehydroxylation and polymerization are likely dependent on the nature of the specific catalytic sites.

The significance of arenol dehydroxylation in coal thermolysis is suggested by two observations. First, Solomon et al. have found that coals begin to lose the major quantity 
of their thermally lost oxygen as water at $350^{\circ}-400^{\circ} \mathrm{C}$, and strikingly the loss is coincident with the release of tars (1990). Second, as demonstrated by our data in Table 1, O-loss is independent of the presence or absence of added water, and thus in the hydrothermal case takes place at about 160 atm of water. The action is therefore essentially irreversible and cannot involve weakly bound nor sorbed molecular water, nor the dehydration of carboxylic acids. Rather water formation must involve, specifically, the elimination of chemically bound elements of water in the organic phase.

The arenol chemistry therefore is the source of both tar and thermal water, and the basis of their concurrent formation. The term "coal-tar" in the scheme emphasizes the fact that in the absence of an aqueous medium (or as in the work by Solomon et al. a flowing gas), the tar is deposited on, or weakly bound to, the coal. It may then engage in subsequent thermally promoted, regressive chemistry, irreversibly reincorporating some fraction of the material back into the coal matrix.

Hydrothermal Treatment. Most of the tar in this case is fully separated from the coal, as shown in the scheme. Given the very high solubility of arenes (Schneider, and Jockers, 1978) and alkanes (Skripka, 1979) in water above $300^{\circ} \mathrm{C}$, it is likely that at hydrothermal conditions the coal is highly swelled and the thermolytic products are virtually fully in solution. Thus with water present the fragments can readily migrate from the coal matrix. It should be noted that we found the tars to be very strongly adhered to the quartz walls. Since quartz surfaces can exhibit considerable acidity, it is of interest therefore to consider the possibility that the tars were generated from the fragment precursors at the walls themselves. This is an aspect of coal chemistry worthy of additional study.

It is worthwhile reiterating the nature of the tars, discussed initially in our second quarterly report (Ross, et al., 1990b). We found the arenol content of the $30 \mathrm{~min}$ tar (by FIMS) to be less than $30 \%$ that of the treated coals. The arenols in the $5 \mathrm{hr}$ tar were reduced yet further by another factor of 3 . $^{*}$ The weight average molecular weights of the two tars were about the same, $393 \mathrm{amu} / 30 \mathrm{~min}$ and $432 \mathrm{amu} / 5 \mathrm{hr}$, but the prolonged hydrothermal treatment lowered the $\mathrm{T}_{1 / 2}$ value from $195^{\circ}$ to $140^{\circ} \mathrm{C}$. Thus the continued hot, aqueous condition, possibly in concert with chemical activity on the quartz surfaces, substantially increases the volatility of the tar, as shown in the scheme.

\footnotetext{
* The arenol content is the sum of the FIMS signals for phenol, dihydroxybenzene, and their respective $\mathrm{C}_{1^{-}}, \mathrm{C}_{2^{-}}$, and $\mathrm{C}_{3^{-}}$derivatives.
} 
Treatment in Undecane. Neither water nor alkanes are good coal swelling media at ambient temperatures, the former because it is too polar, and the latter because it is not polar enough. However water becomes increasingly more coal-accommodating in the hydrothermal region because its dielectric constant decreases to values like those for polar organic liquids (Schneider and Jockers, 1978). Undecane in contrast likely becomes an even poorer solvent, or in effect an antisolvent. The coal should thus not swell but possibly even collapse, and thereby hinder the migration of thermally generated fragments.

As a consequence the arenols will increasingly react within the cage, as depicted in the sequence

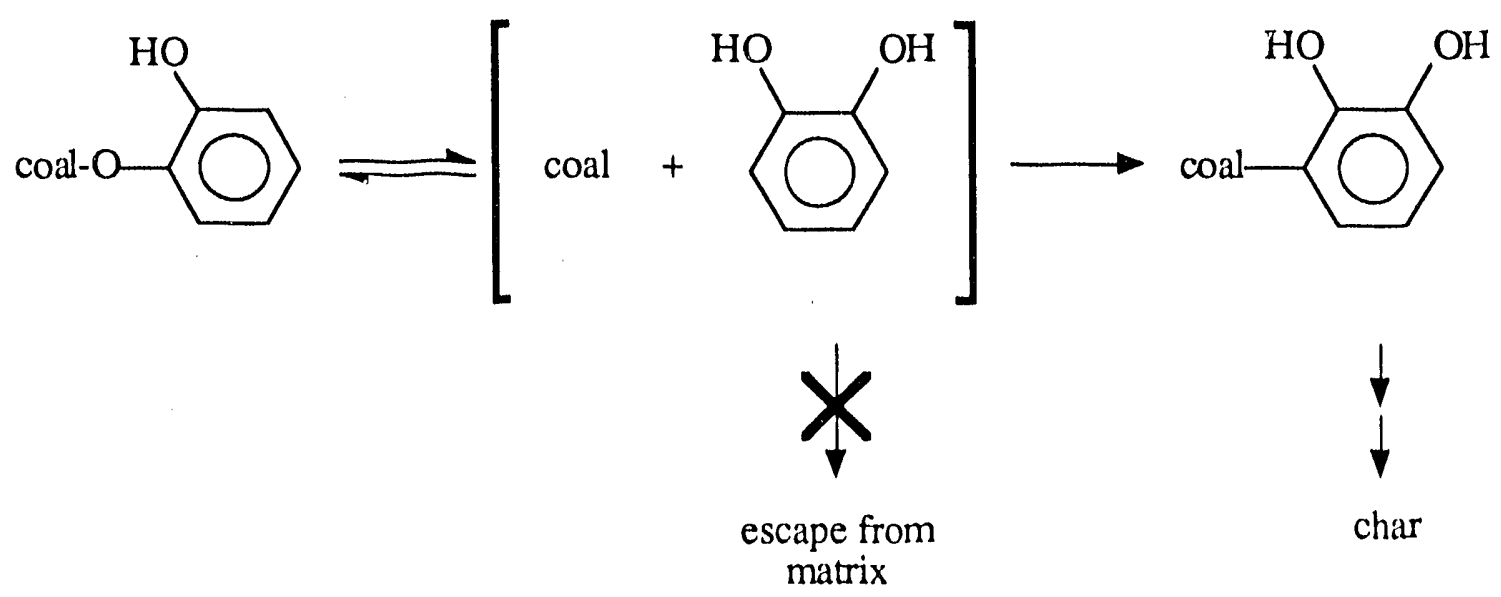

The net result is the replacement of labile aryl ether links by stable $\mathrm{C}$-aryl bonds, which leads in turn to the accumulation of structures that will tend to condense with further heating to char.

Such alkane-promoted condensation chemistry is clearly an undesirable component in conversion generally. Its regressive action, however, could be most seriously encountered in coprocessing, and additional studies in this area could prove profitable.

\section{REFERENCES}

Allen, R. M. and VanderSande, J. B. (1984). Fuel 1984, 3, 24-29.

Lydersen, A. L. (1955). "Estimation of Critical Properties of Organic Compounds," College of Engineering, University of Wisconsin, Eng. Expt. Sta. Rept. 3, Madison, WI.

Malhotra, R., Baker, J. R., Haynes, Jr., H. W., and McCormick, R. L.(1988). Energy and Fuels 1988, 2, 740-743. 
Ross, D. S., and Loo, B, (1990a). Hydrothermal Pretreatrnent of Coal, DOE Contract No. DE-AC22-89PC89880, SRI Quarterly Report No. 3, 30 July.

Ross, D. S., and Loo, B, (1990b). Hydrothermal Pretreatment of Coal, DOE Contract No. DE-AC22-89PC89880, SRI Quarterly Report No. 2, 30 May.

Schneider, G. M. and Jockers, M. (1978). Ber. Bunsenges. Phy s. Chem.1978, 82, 576582.

Skripka, V. G. (1979). Chemistry and Technology of Fuels and Oils 1979, 15, 88-90.

Solomon, P. R., Serio, M. A., Despande, G. V., and Kroo, E. (1990). Energy and Fuels, 4, 42-54 (1990).

Stein, S., Wang, F. M., and Senthilnathan, V. P. (1989). In Proceedings of the 1989

International Conference on Coal Science, (International Energy Agency), pp.165-168. 

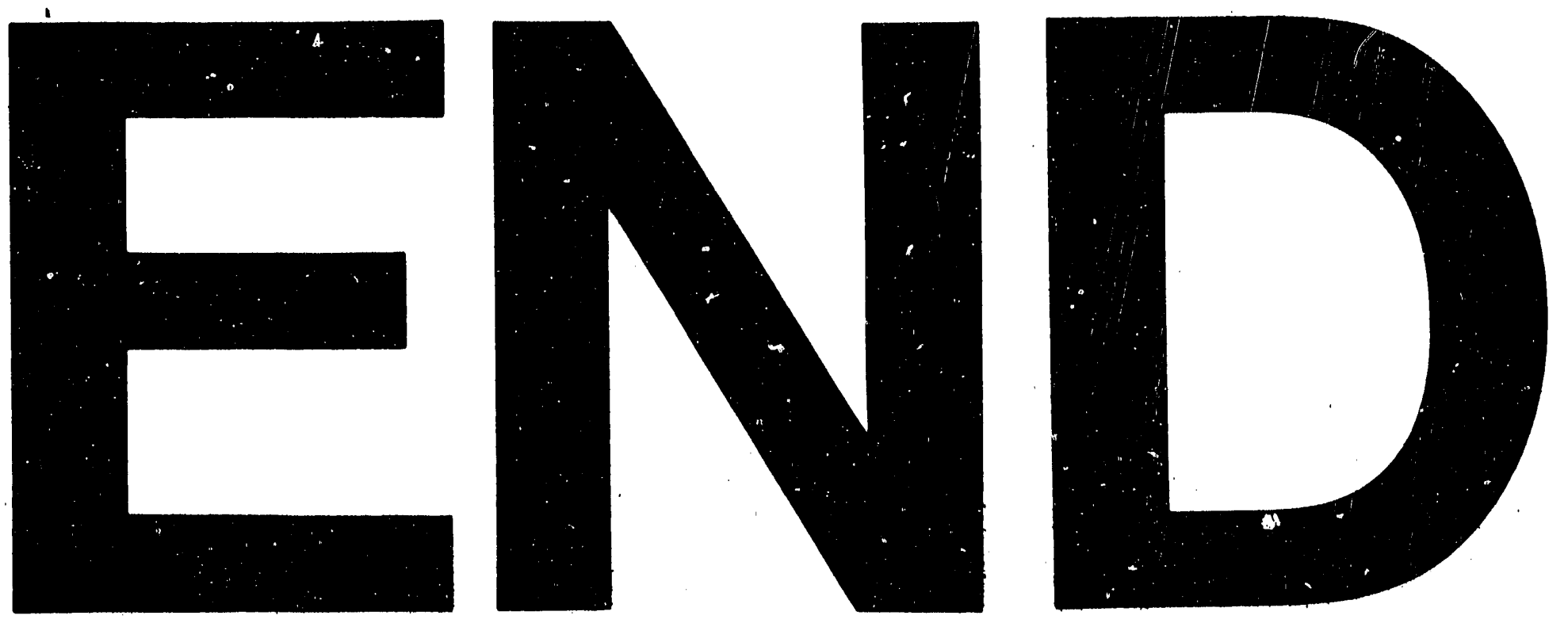

$10 x y=8 x+4$
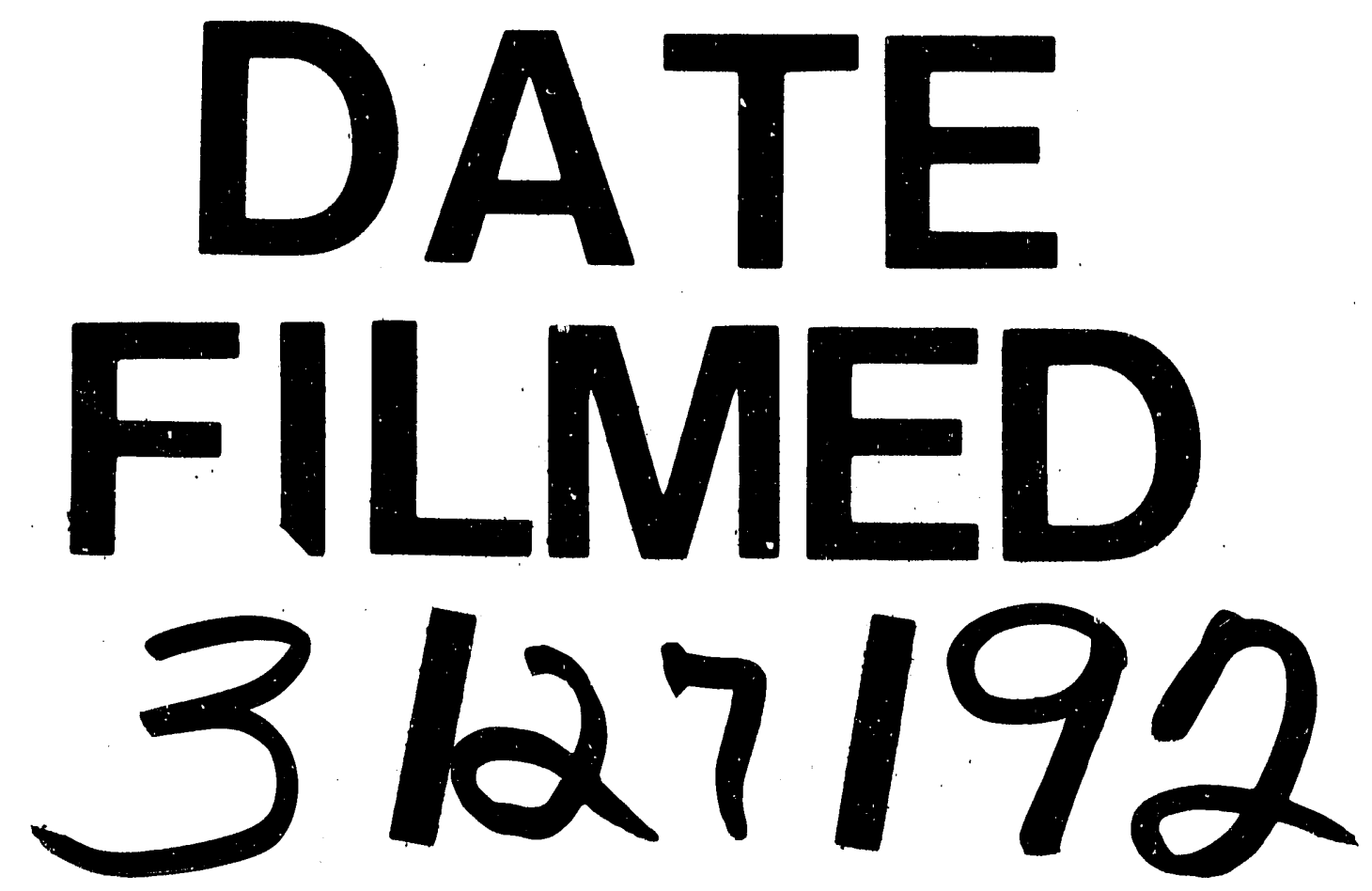
